DOI: https://doi.org/10.46630/phm.12.2020.18

Ivana M. Miljković ${ }^{1}$

Article de recherche

Université de Niš

УДК 81'373.612.2

Faculté de philosophie

Reçu : le 31/1/2020

Département de langue et de littérature françaises

\title{
ANALYSE COMPARATIVE DE LA MÉTAPHORE ET DE LA MÉTONYMIE ET THÉORIES COGNITIVES ASSOCIÉES
}

Nous nous proposons de présenter notre point de vue concernant la métaphore et la métonymie. Éléments de la rhétorique, que Dumarsais définit comme « une espèce de figure », les tropes auxquels nous nous intéressons se voient aujourd'hui dotés de caractéristiques essentiellement linguistiques.

Nous abordons deux aspects.

D'un côté, nous partons des explications de la métaphore en introduisant un terme nouveau que nous avons appelé « la compréhension acquise de la métaphore », qui permet d'expliquer l'existence d'une compréhension partagée.

De l'autre côté, nous examinons les lexèmes à sens multiples qui relèvent de la polysémie. De nos jours, la polysémie ne se limite plus aux lexèmes polysèmes, elle examine aussi d'autres formes du sens multiple, par le biais de la polysémie logique, de la théorie des facettes et des zones actives. L'objectif de cet article n'est pas de faire une présentation minutieuse des théories mentionnées, mais d'établir une liaison entre ces théories d'une part et la métaphore et la métonymie d'autre part.

Vu que c'est la polysémie qui permet la compréhension de la métaphore et de la métonymie, en déclenchant des processus cognitifs spécifiques, nous discutons particulièrement les exemples de Langacker, de Kleiber et de Cruse. Ces linguistes examinent la polysémie dans le cadre de la cognition. Ainsi, nous approchons-nous des sciences cognitives, et de leur terminologie.

Mots clés : métaphore, métonymie, cognition, lexèmes à sens multiples

\section{Introduction}

Les figures de style sont perçues comme des éléments pertinents de la langue déjà à l'époque antique. Depuis Aristote jusqu'à Dumarsais et Fontanier elles sont abordées et expliquées presque systématiquement comme des figures littéraires et oratoires.

${ }^{1}$ ivana.miljkovic@filfak.ni.ac.rs 
Avec le développement de la linguistique, les figures de style, notamment la métaphore et la métonymie, reçoivent plus d'importance, étant analysées par les sémanticiens.

Il existe plusieurs classifications de figures de style. D'après la classification de Mounin (MOUNIN 2000) les figures de style peuvent être des figures de diction telles que l'assonance ou l'allitération; des figures de construction, telle que l'anaphore ou l'épiphore ; des figures de pensée, comme le paradoxe ou des figures de signification, parmi lesquels les plus importants sont la métaphore, la métonymie et la synecdoque.

Les figures de signification ou tropes, consistent à modifier le sens des mots soit par l'analogie qui existe entre deux objets, soit par un rapport d'équivalence.

Dumarsais explique les tropes : «... Les Tropes ne sont qu'une espèce de figure. » (DUMARSAIS 1988,) alors que, quelques siècles plus tard, Joëlle Gardes-Tamine donne sa définition : «...Si c'est le sens des mots qui est l'objet de la figure, on parle de figures de signification, ou tropes. » (GARDES-TAMINE 1996). Jakobson, dans son Essais de linguistique générale (JAKOBSON 1963) ne mentionne que le couple métaphore / métonymie. Les études de Jakobson nous ont été utiles pour établir une liaison entre le processus cognitif et la compréhension des tropes, vu qu'il traite des malades aphasiques.

Nous nous intéressons particulièrement à la métaphore et à la métonymie, en exploitant à la fois les travaux des auteurs mentionnés ci-dessus et des sources littéraires classiques.

\section{Les études de Jakobson}

D'après Jakobson, il existe deux types de patients aphasiques : ceux qui ont perdu la possibilité d'établir le rapport de similarité, et ceux qui ne sont pas capables d'établir le rapport de contiguïté. Les premiers ne sont pas capables de comprendre la métaphore, tandis que les seconds ne peuvent pas saisir la métonymie : «Toute forme de trouble aphasique consiste en quelque altération, plus ou moins grave, soit de la faculté de sélection et de substitution, soit de celle de combinaison et de contexture. La première affection comporte une détérioration des opérations métalinguistiques, tandis que la seconde altère le pouvoir de maintenir la hiérarchie des unités linguistiques. La relation de similarité est supprimée dans le premier type et celle de contiguïté dans le second. La métaphore devient impossible dans le trouble de la similarité et la métonymie dans le trouble de contiguïté. » (JAKOBSON 1963 : 57).

Ces études de Jakobson nous ont mise sur le chemin d'une analyse de l'organisation cognitive de la métaphore et de la métonymie. En effet, si le 
patient aphasique n'est pas capable d'établir la liaison de similarité, il n'est pas capable de sortir d'un champ sémantique afin de créer une idée différente mais correspondante dans l'autre champ sémantique. En revanche, si le patient aphasique n'est pas capable d'établir la liaison de contiguité, il lui est impossible de suivre un lien logique, autrement dit, de faire un glissement référentiel.

Dans le même sens, Le Guern conclut que « le processus métaphorique concerne l'organisation sémique, alors que le processus métonymique ne modifierait que la relation référentielle.» (LE GUERN 1973 : 14). Il est évident que le glissement de sens n'est pas le même dans la métaphore et dans la métonymie. Dans le premier cas, il s'agit d'une image qu'il faut trouver pour comprendre la métaphore, tandis que dans le deuxième cas on reste dans le même champ lexical, étant donné que le glissement de sens se trouve sur le même axe associatif. Le mécanisme métaphorique détruit l'image de la notion habituellement désignée. Le mécanisme métonymique, en revanche, ne la détruit pas, vu que par sa nature la métonymie ne crée pas une image. C'est la relation référentielle qui doit être modifiée, toujours en raison de l'organisation de la métonymie.

\section{Les études cognitives}

En parlant des processus de l'organisation sémique dans la compréhension des tropes, il est utile de répéter que c'est la polysémie qui permet cette compréhension. Lorsqu'on parle de la polysémie, il faut dire qu'il existe plusieurs formes de polysémie (restriction de sens, extension de sens, polysémie étroite et polysémie lâche) (MILJKOVIĆ 2018a).

$\mathrm{Au}$-delà des formes de polysémie, nous sommes témoins de la naissance d'une nouvelle approche de la sémantique, qui, en opérant avec des notions prises des sciences cognitives, se dit « sémantique cognitive ». Dans cette mouvance, la polysémie s'intéresse au processus mentaux mobilisés pour une bonne interprétation de la polysémie logique, ainsi qu'à différentes théories qui traitent le sens multiple.

D'après Rastier, « la sémantique cognitive prend pour principe que le sens linguistique consiste en représentation ou processus mentaux, ce qui la conduit à s'absorber soit dans la psychologie, soit dans une philosophie spontanée. [...]. Le domaine de l'espace, privilégié par la sémantique cognitive, en offre une illustration qui éclaire ses rapports avec l'esthétique transcendantale. » (RASTIER 1993: 153, 154). La polysémie logique se situe dans le cadre de la sémantique cognitive. Sans entrer dans l'esthétique transcendantale, ni dans la philosophie de Kant, nous retenons ici seulement le « principe [qui mobilise les] processus mentaux ». 
C'est à partir de ce principe que nous allons expliquer la compréhension des tropes, dans notre cas la métaphore et la métonymie. Les processus mentaux donnent la capacité d'appréhender les notions abstraites et par conséquent la capacité à comprendre la catégorisation des tropes. D'ailleurs, nous examinons ici la métaphore et la métonymie du seul point de vue linguistique, en négligeant l'angle rhétorique pour rester sur celui de la sémantique cognitive.

Dans le cadre du cognitivisme, nous utilisons certains termes empruntés à la psychologie, comme gestalt ou à la philosophie lorsqu'on parle de la métaphore conceptuelle, ou encore à la logique pour mieux éclaircir la différence entre la polysémie proprement dite et la polysémie logique.

Pour autant, notre discours sur la compréhension acquise de la métaphore - concept que nous introduisons plus bas - est basé sur une présentation plus traditionnelle des tropes.

\section{La métaphore}

Meyer dit pour la métaphore : «La métaphore est la substitution identitaire par excellence, puisqu'elle affirme que A est B. » (MEYER 2008 : 71)

Cette affirmation est, d'après nous, une réflexion juste sur la métaphore. Lorsque Doña Sol dit à Hernani : " Vous êtes mon lion superbe et généreux » (HUGO 1830 : Hernani), elle l'identifie au lion et affirme que A (Hernani) est B (lion). Une substitution par excellence, en effet, puisqu'un être humain est vu comme un animal. Et c'est justement cette substitution qui pousse le lecteur à chercher la bonne compréhension.

Ladite compréhension va mettre en œuvre des processus mentaux, à l'aide desquels le lecteur va trouver l'image correspondante à cette métaphore.

Lakoff et Johnson s'opposent à l'idée que la métaphore a seulement un sens figuré. D'après eux la métaphore elle-même représente un concept, et on peut parler de la métaphore seulement si l'on est capable de comprendre un aspect de la réalité en le comprenant en d'autres termes. (LAKOFF, JOHNSON 1986). La métaphore influence notre pensée et se trouve à la base de nombreux concepts de la pensée humaine.

Ceci nous rapproche du champ de la cognition, qui introduit un type de métaphore qui lui convient : la métaphore conceptuelle : " Pour souligner l'appartenance de la métaphore à la pensée, on introduit la notion de métaphore conceptuelle. Le mécanisme de la métaphore conceptuelle consiste à cartographier la structure d'un concept (ou domaine conceptuel), qui est généralement une perception connaissable et bien connue, dans un autre domaine. [...] Autrement dit, nous comprenons les domaines abstraits 
en fonction des expériences que nous avons avec le monde physique. Pour cette raison, les métaphores conceptuelles constituent les principaux moyens à notre disposition pour comprendre des expériences abstraites. » (JOVANOVIĆ 2015).

Le fait de sortir d'un champ sémantique pour aller dans un autre, dans lequel on exploite des sémèmes en commun donne l'impression qu'on fait une forme de comparaison (Hernani est comme un lion). Ceci a conduit à une explication erronée selon laquelle la métaphore serait une « comparaison abrégée », comme l'affirme Quintilien (1875).

\subsection{Une erreur venant de loin : confusion entre comparaison et similitude}

On est en présence d'une confusion qui remonte à l'antiquité, au moment où la comparaison et la similitude n'étaient pas bien distinguées. D'ailleurs, il s'agit d'une erreur qui s'est retrouvée apparemment dans plusieurs langues. Les deux mots latins, " comparatio » qui veut dire " comparaison » et « similitudo » qui veut dire « similitude » sont, dans la terminologie française, traduits par un même mot, celui de " comparaison ». Or, il s'agit de deux concepts différents, puisque dans comparatio on a deux éléments comparables sur une même échelle, alors que dans similitudo on ne compare pas les éléments en question, on cherche les traits communs qui les mettent dans une relation de similitude, par leurs propriétés partagées.

Le Guern donne des exemples : " Pierre est aussi fort que son père » et « Pierre est fort comme un lion » (LE GUERN 1973). La première phrase peut être interprétée «Pierre est fort comme son père ». En revanche, la phrase «Pierre est fort comme un lion » ne peut pas être interprétée de la même façon, à savoir « Pierre est aussi fort qu'un lion ». Dans le premier cas il s'agit d'une comparaison de deux éléments comparables. Dans le deuxième cas, « le lion » est la référence de la force. Le deuxième exemple veut dire que Pierre est très fort, mais comparer sa force avec la force réelle d'un lion serait exagéré. Le premier exemple est une véritable comparaison. Le deuxième exemple représente similitudo, puisque le second argument sort de la comparaison et est aperçu comme l'image de la force.

Une fois cette ambiguïté résolue, nous nous rendons compte que toutes les liaisons entre la métaphore et la comparaison deviennent discutables. On a du mal à trouver des comparaisons qui - en enlevant le mot « comme »deviennent des métaphores, de même que des métaphores que l'on pourrait transformer en comparaison. Nous avons l'impression que la plupart des métaphores avec la copule peut se transformer non pas en comparaison, mais en similitude, comme dans les exemples : « La nature est un temple » ce qui donnerait la transformation « La nature est (divine, riche, sacrée, etc.) 
comme un temple »; «Achille est un lion » ce qui donnerait la transformation «Achille est fort comme un lion ».

Dans la même logique, nous pensons que la phrase d'Éluard « La Terre est bleue comme une orange » (ÉLUARD 1929: L'amour la poésie) correspond, non pas à une comparaison mais à une similitude, qui peut se transformer en la métaphore : « La terre est une orange bleue ».

\subsection{Notre explication de la métaphore}

Touratier examine seulement la métaphore avec la copule : «En réalité, s'il y a métaphore, c'est à la fois parce qu'il n'y a rien de commun entre les sémèmes des termes mis en rapport syntaxique et par conséquent sémantique par la copule être, et parce que ce rapport syntaxique oblige l'interlocuteur à construire ou imaginer un trait sémique commun, en fonction du contexte, de la situation référentielle désignée par les sémèmes en question, et aussi, bien entendu, de la situation énonciative dans laquelle sont employés ces deux sémèmes. 》 (TOURATIER 2000 : 78-79).

Cependant, nous pensons que la véritable nature de la métaphore peut se révéler non seulement dans la métaphore avec la copule, mais surtout dans les métaphores ayant un verbe autre que la copule, cas dans lequel la transformation en comparaison/similitude n'est pas possible, et qui, par conséquent, illustre le cas dans lequel la métaphore ne peut pas être considérée comme comparaison ${ }^{2}$ abrégée. Parmi les métaphores qui ne peuvent pas subir cette transformation, les exemples sont nombreux : «Les vagues jappent », « La forêt a mangé le ciel », « les mots brûlent la gorge », etc.

\subsection{La compréhension acquise de la métaphore}

La puissance de la métaphore se trouve dans le fait qu'elle crée une image. Nous prétendons que chacun peut associer sa propre image à une même métaphore ce qui conduit à différentes possibilités de compréhension d'une même métaphore.

La métaphore lexicale «Achille est un lion » nous sert comme exemple pour un terme nouveau que nous expliquons ci-dessous.

«Achille est un lion » veut associer à Achille des qualités du lion. On pourrait penser aux qualités de férocité, en pensant à Achille lorsqu'il traîne le corps d'Hector ; ou de noblesse, Achille étant fils du roi Pélée, ou de force, vu qu'il est considéré comme le plus grand héro grec.

Pourtant, nous pouvons constater que la plupart des gens comprennent la métaphore «Achille est un lion » comme « Achille est courageux comme un lion $»$. Nous nous trouvons donc devant un paradoxe puisque nous affirmons

${ }^{2}$ Sous le terme « comparaison » nous sous-entendons à la fois comparatio et similitudo. 
que la compréhension métaphorique peut être individuelle, vu que chacun peut se créer sa propre image de la métaphore ; et que cependant il est avéré que tous les récepteurs comprennent une métaphore donnée - au moins dans certains cas - d'une seule et unique manière. Pour résoudre ce paradoxe qui n'existe, nous semble-t-il, qu'en apparence, nous nous proposons d'introduire un terme par lequel il nous serait possible de résoudre ce problème. Nous allons appeler ce terme « la compréhension acquise de la métaphore ». Quasiment tout le monde compare le héros grec au lion pour son courage physique. On a appris à voir un lion en Achille reconnaissant son courage physique. La même chose se produit avec d'autres métaphores - en serbe ou en français - surtout pour les métaphores types ${ }^{3}$ : elles sont souvent comprises de la même manière non pas parce que d'autres manières n'existent pas, mais souvent parce que c'est la manière dont on a appris à comprendre les choses.

Cette manière de comprendre les choses dépend de la culture, de la population et des connaissances collectives caractéristiques de cette population.

À part l'exemple donné, nous allons citer des exemples des chants populaires pris de la littérature orale serbe, dans lesquelles la métaphore est très répandue. Soit l'exemple : "Ses yeux sont deux pierres précieuses » ou un autre : "Ses dents sont deux colliers de perles », tels que rapportés par Karadžić dans le chant populaire « Le mariage de Milić porte-drapeau » (KARADŽIĆ 1845).

Dans les chants populaires, les métaphores citées sont typiques pour décrire la beauté d'une fille. Il est évident que dans le premier exemple personne ne va comprendre les deux pierres précieuses comme deux diamants ou deux rubis, rarement comme deux saphirs. Dans la plupart de cas le lecteur comprend qu'il s'agit des émeraudes, à cause de la couleur. En revanche, les dents ne sont pas précieuses comme des perles, elles sont blanches et bien rangées, comme un collier de perles taillées. Pourtant, d'autres possibilités de compréhension existent : la fille en question peut avoir le regard clair comme les diamants et les dents irrégulières comme des perles brutes. Cependant, tous les récepteurs comprennent qu'il s'agit d'une belle fille aux yeux verts et aux dents régulières.

Nous expliquons ceci par la compréhension acquise de la métaphore, vu qu'ellen'est pas conditionnée seulement par les faits liés au milieu socioculturel, mais aussi par le fait que les récepteurs ont déjà une connaissance acquise qui les pousse à créer systématiquement une même image. Nous croyons que ceci arrive à cause du fait que tous les récepteurs ont appris à comprendre certains concepts suivant le même mécanisme.

${ }^{3}$ Lorsque nous parlons de la métaphore type, nous sous-entendons notamment la métaphore morte. 


\section{La métonymie}

Touratier, dans sa Sémantique, explique ainsi le processus métonymique : "Avec la métonymie, le sémème ne change pas à proprement parler de contenu. [...] Le lexème ne change pas alors de sémème, mais il change de référent. Son sémème désigne non pas son référent usuel, mais un référent qui est objectivement ou culturellement lié à ce référent usuel, et qui correspond normalement au sémème d'un autre lexème » (TOURATIER $2000:$ 75).

La différence entre la métonymie et la métaphore est évidente. Puisqu'il y a contigüité dans la liaison logique, il est beaucoup plus facile pour le récepteur de parvenir à la compréhension. On reste dans le même champ sémantique, avec simplement un glissement référentiel. Les différentes formes de métonymie correspondent à différents glissements de sens, mais dans tous les cas la liaison est claire et non ambigüe, comme dans les exemples « le vin est bon ; j'ai bu trois verres » ou « Phèdre a bu la mort».

Le processus mental utilisé pour la compréhension de la métonymie est plus simple que celui utilisé pour la métaphore. La raison en est simple : au lieu de changer le champ sémantique, il suffit de faire le glissement référentiel. Cependant, c'est la métonymie qui se trouve, dans la plupart des cas, au sein des recherches sémantiques, notamment dans le cadre de la polysémie logique (Cruse (1986) Kleiber (1999), Langacker (1984)). C'est à partir de la métonymie qu'il est possible d'analyser les lexèmes à plusieurs sens, qu'il s'agisse des facettes, des zones actives, ou d'une autre forme de polysémie. Il s'est avéré que la métaphore, dont la compréhension exige le changement du champ sémantique laisse peu de place à l'étude des lexèmes à sens multiples.

Nous nous sommes limitée au couple métaphore/métonymie, en suivant la classification de Jakobson. Pour lui, la synecdoque représente, à juste titre, une forme de métonymie. C'est à partir de cette distinction que Kleiber explique la métonymie intégrée, même s'il parle du rapport de tout pour la partie et inversement.

D'après Dumarsais (1988), qui considère que la synecdoque est une figure à part, il existe neuf formes de métonymie : la cause pour l'effet ; l'effet pour la cause ; le contenant pour le contenu; le nom du lieu où une chose se fait, se prend pour la chose même ; le signe pour la chose signifiée ; le nom abstrait pour le concret ; les parties du corps qui sont regardées comme le siège des passions et des sentiments intérieurs, se prennent pour les sentiments mêmes ; le nom du maître de la maison se prend pour la maison qu'il occupe.

En examinant de près les formes métonymiques traditionnelles, nous constatons que la plupart d'entre elles d'elles aujourd'hui reste dans le domaine littéraire et que, pour l'approche qui nous intéresse ici, toute forme de métonymie peut se réduire au simple glissement référentiel. De même, 
l'attribution actuelle de la synecdoque à la métonymie est faite à juste titre, notamment à cause de son rôle dans les recherches polysémiques que nous abordons ci-dessous.

\subsection{La nature cognitive de la métonymie}

D’après Kleiber (1999a : 132) « si la déviance métaphorique ne donne pas lieu à une lecture métonymique ou synecdotique, c'est parce que la déviance n'est pas identique, même si dans les deux cas, il s'agit d'un emploi catégoriel indu. Dans le cas de la métonymie/synecdoque, l'emploi abusif résulte de l'utilisation de la catégorie d'une occurrence pour une autre occurrence d'une catégorie incompatible, alors que dans la métaphore, il s'agit de la catégorisation d'une occurrence dans la catégorie qui ne lui est normalement pas destinée. »

Cela exprime le fait que dans une métonymie, le changement de catégorie associe deux catégories incompatibles, sans sortir du champ sémantique.

Dans ses explications, Antin Fougner Rydning (2003) se concentre surtout sur le rôle cognitif de la métaphore et de la métonymie. Pour expliquer la métonymie, elle fait appel aux cognitivistes et propose une approche dans cet esprit : « Les cognitivistes, qui ont emprunté le terme de métonymie à la rhétorique, lui confère un rôle bien plus étendu que celui de trope. Outre le fait que la métonymie est un procédé référentiel, ils voient en elle un mécanisme général de la compréhension du sens. La métonymie est présente dans tous les textes, quels qu'ils soient, non pas de façon aléatoire et occasionnelle, mais de façon régulière et constante. [...] La métonymie n'est pas un phénomène exclusivement linguistique, elle fait partie intégrante des systèmes conceptuels qui sous-tendent notre façon de penser et d'agir. Fondée sur notre expérience, la métonymie conceptuelle est un processus de la pensée qui permet d'accéder à la compréhension. »

Tout en insistant sur le fait que « la métonymie est un processus cognitif [...] qui met en œuvre la contiguité [...] dont la nature est conceptuelle, car la métonymie fait appel à une représentation mentale où le sujet mobilise ses connaissances extralinguistiques », Antin Fougner Rydning examine la différence entre la métonymie et la métaphore.

En effet, elle part des études de Gibbs (1997) qui pense qu'il est difficile parfois de faire une distinction nette entre la métaphore et la métonymie, et qui propose un test de similitude pour faire la différence. Antin Fougner Rydning donne les exemples suivants : «I am a one man's dog » et « Le sandwich au jambon attend l'addition. » Ensuite elle applique le test de similitude de Gibbs en disant que, puisqu'il est possible de dire "I am like one man's dog », il s'agit de métaphore; en revanche, «Le sandwich au jambon est comme une personne qui attend l'addition. » n'a pas de sens et ne peut donc relever de la métaphore. On est en conséquence en présence de métonymie. 
Patrick Saint-Dizier donne sa définition informelle dans un article sur la métonymie : « La métonymie est une opération linguistique et cognitive qui a essentiellement une fonction référentielle, en ce qu'elle autorise l'emploi d'une entité pour en représenter une autre. Il doit exister une relation entre l'entité utilisée et celle référencée. Cette relation est essentiellement de deux types : la relation paradigmatique partie-tout (employée en partie vers le tout ou en tout vers la partie) et un ensemble a priori ouvert de relations fonctionnelles. » (SAINT-DIZIER 2006).

Ce que nous retenons de l'article de Saint-Dizier est l'énoncé tropique qui peut être à la fois un énoncé métaphorique et métonymique, comme dans l'exemple suivant : «L'Europe croit à la démocratie », dans lequel nous avons d'abord une métaphore (une entité géographique vue comme un humain), ensuite une métonymie (les habitants de l'Europe croient à la démocratie). Cet exemple de Saint-Dizier, et son explication métaphorique qui se base sur la personnification illustre le fait que la personnification est une figure appartenant au trope métaphorique.

\subsection{Autres approches de la compréhension cognitive de la métonymie}

\section{A) Les zones actives}

En 1984 Ronald Langacker (1984 : 172-188) présente sa théorie des zones actives dans l'article intitulé Active Zones. Cette théorie traite de l'usage de substantifs globaux en lieu et place de substantifs n'en désignant qu'une partie, un effet, un attribut... nommé zone active.

Dans ladite théorie, l'auteur s'intéresse notamment de la relation prédicative entre l'argument sujet et l'argument objet. Langacker introduit les termes de « trajecteur " pour l'acteur principal et de «landmark » pour l'acteur secondaire.

En 1999 dans Problèmes de sémantique, Kleiber (1999b) reprend cette théorie et s'exprime à son tour.

La théorie est présentée en donnant notamment les exemples suivants : « David blinked »; " She heard the piano »; «I'm in the phone book », commentés avec humour en disant que ce n'est pas la personne entière de David qui fait clin d'œil ; qu'on ne peut pas entendre un objet et finalement, que la personne, pour être dans l'annuaire doit être soit très petite, soit à l'étroit.

L'exemple le plus illustratif pour les zones actives est, nous semble-t-il, le premier : c'est l'œil qui est actif, pas la personne en totalité. Néanmoins, nous sommes obligée de constater que cet exemple est représentatif en anglais et en serbe, mais pas en français. Ceci s'explique par le fait que le landmark, qui normalement n'est pas exprimé dans la phrase, fait partie de l'expression française «faire un clin d'œil». Cependant, nous pouvons citer pour le français l'exemple que Kleiber a repris de Langacker : " Roger mange une pomme. » 
Les autres exemples que Kleiber reprend pour présenter la théorie en question sont les suivants : "Votre chien est près de mon chien "; "Votre chien a mordu mon chat ». Les zones qui sont établies sont celles qui activement participent aux processus. Dans le premier cas, les animaux dans leur globalité ; dans le deuxième cas la mâchoire du chien et une partie du chat.

On peut faire un lien entre les zones actives et la métonymie. Ce lien s'explique, comme Kleiber le souligne, par la théorie de gestalt. En effet, en évoquant le chien, on imagine en fait sa mâchoire mordant le chat, autrement dit, nous assimilons automatiquement la forme globale à l'élément constitutif actif dans l'action de mordre. Tous les exemples qui illustrent les zones actives sont de nature métonymique.

C'est grâce à la métonymie qu'on est capable de comprendre les exemples de Langacker. La relation prédicative est souvent décrite de façon incomplète, comme dans les exemples « Ce stylo rouge est jaune » et «Paul est dans la baignoire ». Dans le premier exemple le texte est ambigu ; on ne sait pas si le stylo est en plastique jaune et écrit en rouge, ou bien si c'est le contraire. Le deuxième exemple illustre les zones actives, vu que ce n'est pas le corps entier de Paul qui est dans la baignoire, sa tête et ses cheveux n'y étant pas, mais en même temps il est possible de l'expliquer par la métonymie intégrée.

Les zones actives ne représentent pas des entités bien identifiées. Au contraire, il est difficile de voir où la zone active commence et où elle prend fin. Les exemples mentionnés en font preuve. Les manifestations desdites zones ne sont pas forcément de nature concrète. L'exemple «Elle entendit le piano » renvoie à « une raison cognitive [...] qui permet [...] de focaliser l'attention sur des entités qui ont une certaine saillance cognitive... » (KLEIBER 1999b). Le rapport entre le piano et le son du piano est bien un rapport de contiguïté, donc de nature métonymique.

B) La théorie des facettes

D'une manière générale, nous ne pouvons pas parler de la polysémie du lexème «fenêtre ». Néanmoins, il y a une différence de sens du mot dans les phrases : 1) Marie a repeint la fenêtre et 2) Paul est sorti par la fenêtre (KLEIBER 1999: 87). Dans le premier cas « la fenêtre » évoque l'encadrement de la fenêtre (en bois par exemple), tandis que dans le deuxième cas on pense à l'ouverture de la pièce qu'est la fenêtre, ce qui renvoie à une entité différente. Cette polysémie logique ouvre le chemin à des recherches plus avancées concernant le sens (MILJKOVIĆ 2018b).

Dans ce cadre, nous nous intéressons à la théorie des facettes qui vient de Cruse (1986) mais que nous présentons par le biais de la présentation française faite par Kleiber (1999). 
Cruse propose de « fournir les moyens nécessaires pour identifier ce concept sémantique nouveau de facette » (KLEIBER 1999 :89).

Nous voyons la théorie des facettes comme un intermédiaire qui permet l'établissement d'une différence entre la polysémie proprement dite et l'analyse des lexèmes à sens multiples. Vu que les facettes ne sont pas perçues comme une caractéristique intrinsèque de la langue, elles permettent la description subtile de sens entre les lexèmes qui ne sont pas en soi polysèmes mais qui néanmoins peuvent avoir une différence dans l'usage.

En pratique, cela veut dire qu'on examine tous les sens possibles dans lesquels un même lexème peut avoir des significations différentes. L'exemple suivant en fait preuve :

La banque dans la grand-rue a brûlé hier soir. [IMMEUBLE]

La banque est très aimable avec moi [PERSONNEL] La banque a été créée en 1900 [INSTITUTION] (KLEIBER 1999 : 93)

En développant cette théorie, Kleiber mentionne quelques éléments : le concept global représente une seule gestalt; dans la dimension verticale de la sémantique de prototype, la place de niveau de base est occupée par le concept global et non par les facettes isolées ; concernant la dimension horizontale, on prend en considération le prototype ou le meilleur exemplaire de l'entité en question.

Il nous semble que l'élément qui fait la meilleure distinction entre la polysémie proprement dite et les facettes se trouve dans le fait que les lexèmes polysèmes sont en compétition, alors que les facettes ne le sont pas. De même, nous soulignons encore une fois la différence principale qui se trouve dans la nature de la langue. Alors que la polysémie est perçue comme une caractéristique intrinsèque de la langue, les facettes ne le sont pas.

Les facettes, au même titre que les zones actives et la métonymie intégrée, relèvent d'une approche cognitive originale qui vient enrichir les analyses qu'on peut faire des lexèmes à sens multiples.

\section{C) La métonymie intégrée}

Il existe des lexèmes dont la nature polysémique n'est pas évidente. Le lexème « visage » désigne habituellement la face de la tête. Pourtant, on trouve des phrases comme « Le nouveau visage de la France, terre d'immigration » (titre d'un article du Monde). Dans cet exemple « visage » renvoie à l'image de la France comme terre d'accueil. Le sens n'est pas le même dans des exemples « visage de la France » et « le joli visage d'une fille». Il s'agit d'un lexème à sens multiples qui peut être analysé par différentes approches. Lorsqu'il s'agit de ces deux exemples, nous pouvons parler des facettes du lexème " visage ".

Restant sur le concept de visage, une autre problématique se pose. Prenons l'exemple : «Anna est maquillée ». On comprend bien que c'est 
seulement le visage d'Anna qui est maquillé et non pas son corps entier. La phrase est comprise de façon adéquate, et cela peut s'expliquer par le principe de la métonymie intégrée.

Le principe de la métonymie intégrée est : « Certaines caractéristiques de certaines parties peuvent caractériser le tout ( (KLEIBER 1999 :143) comme illustré par ses exemples : Les américains ont débarqué sur la lune en 1969 ; Le pantalon est sale, etc.

Les deux exemples de Kleiber suffisent à illustrer ledit principe. Il suffit qu'une tache figure sur le pantalon, pour dire que le pantalon est sale, et pourtant toute la surface du pantalon n'est pas sale, aussi bien que tous les Américains n'ont pas aluni. Il s'agit de la propriété du prédicat qui, sans avoir besoin d'être vérifié sur une partie ou toute l'entité est vrai : " [le principe de la métonymie intégrée] vise à rendre compte d'un phénomène cognitif majeur, à savoir qu'un référent, dans un sens très large du terme, peut se voir appliquer des propriétés, événements, etc. qui concernent en fait uniquement certaines de ses « parties ». » (KLEIBER 1999).

C'est ce principe de métonymie intégrée qui permet de comprendre la phrase " Anna est maquillée ».

L'usage de ce principe contribue aussi à l'analyse des lexèmes à sens multiples et en particulier dans le cadre de la métonymie.

\section{Conclusion}

Les figures de style, et notamment les tropes ont été étudiées dès l'antiquité. Les philosophes et les littéraires les ont décrites sous l'angle de la rhétorique ou de la littérature. Cependant, aujourd'hui il est possible d'expliquer les tropes sous l'angle de la linguistique.

Nos recherches, que nous avons faites en utilisant une analyse comparative entre les différentes explications des tropes d'un côté et le rôle important des différentes théories cognitivistes de l'autre côté, nous ont conduite sur deux chemins.

Nous intéressant à l'interprétation de la métaphore dans le cadre traditionnel, nous avons remarqué que, malgré la multiplicité des interprétations possibles d'une même métaphore, la même image était perçue par la plupart des gens. Nous avons voulu comprendre ce phénomène, et nous sommes arrivée à penser qu'il y avait une raison fondée sur une communauté d'histoire, de culture et d'apprentissage, ce que nous avons traduit sous le terme de « compréhension acquise de la métaphore».

Sur un plan différent et complémentaire, nous avons exploré une partie du large champ des études cognitives touchant à la polysémie. 
La compréhension de la métaphore conceptuelle relève de processus mentaux et est ainsi intimement liée aux mécanismes cognitifs. Il s'agit en fait de percevoir, de comprendre, un aspect de la réalité, ce qui nécessite un transfert cognitif de champ sémantique depuis celui textuellement exprimé vers celui de la réalité interprétée.

Les travaux des sémanticiens cognitivistes ont permis un éclairage nouveau surl'étude de la métonymie. La métonymieintégrée permet d'expliquer les glissements référentiels notamment entre « le tout et la partie »; les zones actives permettent d'analyser aussi cet aspect, mais en se focalisant sur les contextes d'action. Toutes deux aident à comprendre comment la métonymie est alors à la fois utile, car simplificatrice, et néanmoins non ambigüe.

La théorie des facettes, de son côté, veut décrire des cas de métonymie où un lexème est utilisé de façons différentes, et s'interprète grâce à un concept implicite complémentaire relevant du contexte. La polysémie ne semble pas alors évidente ; la théorie des facettes aide à l'expliquer.

Les études cognitives sont un outil précieux pour approfondir la subtilité de différentes formes de polysémie qui diffèrent de la polysémie proprement dite.

\section{Bibliographie}

CRUSE 1986 : CRUSE, David Alan. Lexical semantics. Cambridge: Cambridge University Press, 1986.

DUMARSAIS 1988 : DUMARSAIS, César Chesneau. Des tropes ou des différents sens. Paris : Flammarion, 1988.

GARDE-TAMINE 1996 : GARDE-TAMINE, Joëlle. La rhétorique, Paris : Armand Colin, 1996.

GIBBS 1997 : GIBBS, Raymond. Metaphor in Cognitive Linguistics. Amsterdam : John Benjamins publishing company Amsterdam/ Philadelphia : 1997.

JAKOBSON 1963 : JAKOBSON, Roman. Deux aspects du langage et deux types d'aphasie, dans : Essais de linguistique générale. Paris : Éditions de Minuit, 1963.

JOVANOVIĆ 2015 : JOVANOVIĆ, Ivan. Les proverbes français avec les lexème âne et cheval et leurs équivalents serbes. $2015<\mathrm{https}: / /$ etudesslaves.edel.univpoitiers.fr:443/etudesslaves/index.php?id=914> 28. 01. 2020.

KLEIBER 1999a : KLEIBER, Georges. «Espèces d'espaces 》 : approche linguistique et sémiotique de la métaphore, de CHANAY et RÉMIGIRAUD, 2002. < < $\quad<$ ttps://journals.openedition.org/mots/7013>. 05. 04. 2020. 
KLEIBER 1999b : KLEIBER, Georges. Problèmes de sémantique, La polysémie en question, Villeneuve d'Ascq : Presses Universitaires du Septentrion, 1999.

LAKOFF, JOHNSON 1986 : LAKOFF, Georges et JOHNSON, Michael : Les Métaphores dans la vie quotidienne, Paris : Éditions de Minuit, 1986.

LANGACKER 1984 : LANGACKER, Ronald : Proceedings of the Tenth Annual Meeting of the Berkeley Linguistics Society. Berkeley, 1984.

LE GUERN 1973 : LE GUERN, Michel : Sémantique de la métaphore et de la métonymie. Paris : Librairie Larousse, 1973.

MEYER 2008 : MEYER, Michel. Principia rhetorica : Une théorie générale de l'argumentation. Paris : Fayard, 2008.

MILJKOVIĆ 2018a : MILJKOVIĆ, Ivana. "Analyse comparative de quelques cas d'homonymie et de polysémie en français et en serbe ». Nauka i savremeni univerzitet (2018). Niš : 2018.

MILJKOVIĆ 2018b : MILJKOVIĆ, Ivana. « La théorie des facettes appliquée à la langue serbe », Nasleđe (2018). Kragujevac : 2018.

MOUNIN 2000 : MOUNIN, Georges : Dictionnaire de la linguistique. Paris : Quadrige, 2000.

QUINTILIEN 1875 : Institution oratoire, VIII Chapitre VI. Paris : FirminDidot et Cie, 1875.

RASTIER 1993 : RASTIER, François. « La sémantique cognitive. Éléments d'histoire et d'épistémologie ». Histoire Épistémologie Langage, tome 15 p 153-187; <https://www.persee.fr/doc/hel_07508069_1993 num_15_1_2372>.05.04. 2020.

RYDNING 2003 : RYDNING, Antin Fougner, « La métonymie conceptuelle ». Romansk Forum, Nr. 17 - 2003/1. <http://docplayer. me/21155105-Romansk-forum-nr-17-2003.html>. 05. 04. 2020.

SAINT-DIZIER 2006 : SAINT-DIZIER, Patrick : 2006. « Métonymie ». Sémanticlopédie : dictionnaire de sémantique.

$<$ http://www.semantique-gdr.net/dico/index.php/Métonymie>. 05. 04. 2020.

TOURATIER 2000 : TOURATIER, Christian : La sémantique. Paris : Armand Colin, 2000.

\section{Sources}

ÉLUARD 1929 : ÉLUARD, Paul : L’amour la poésie. Paris : Gallimard, 1929.

HUGO 1830 : HUGO, Victor: Hernani, $1830<$ https://data.bnf.fr/fr/11976057/ victor_hugo_hernani/>.05.04. 2020.

KARADŽIĆ 1845: KARADŽIĆ Stefanović Vuk: Le mariage de Milić porte-drapeau, 1845. 
$<$ https://www.delfi.rs/knjige/86852_srpske_narodne_pjesme_iii_knjiga_ delfi_knjizare.html>.05.04.2020.

Ivana M. Miljković

\section{UPOREDNA ANALIZA METAFORE I METONIMIJE UZ PRIKAZ ODGOVARAJUĆIH KOGNITIVNIH TEORIJA}

Predmet našeg interesovanja u ovom radu su metafora i metonimija. Dimarse je trope definisao kao vrstu figura, a mi danas konstatujemo kako su u bliskoj vezi sa lingvistikom. Zato ih obrađujemo sa dva aspekta, prikazujući našu tačku gledišta.

S jedne strane, polazimo od klasičnih objašnjenja tropa, a zatim dajemo naše objašnjenje metafore. Pritom, uvodimo novi termin - jednoobrazno shvatanje metafore - pomoću kojeg možemo objasniti postojanje uvreženog shvatanja metafore.

$\mathrm{S}$ druge strane, proučavamo višeznačne lekseme kojima se bavi polisemija. Uočeno je da se polisemija ne ograničava samo na polisemne lekseme, već se bavi i drugim oblicima višeznačnosti. Navešćemo samo neke oblike: logička polisemija, teorija višeznačnih leksema (Théorie des facettes, v. MILJKOVIĆ 2018b), kao i aktivne zone (LANGAKER 1984).

Budući da se preko polisemije, koja izaziva specifične kognitivne procese, dolazi do razumevanja metafore i metonimije, naročito razmatramo Langakerove, Kleberove i Kruzove primere. Pomenuti lingvisti proučavaju polisemiju u okviru kognitivizma. Na taj način se približavamo kognitivnim naukama i njihovoj terminologiji.

Cilj članka nije detaljno predstavljanje pomenutih teorija, već uspostavljanje veze između tih teorija i metafore i metonimije.

Ključne reči: metafora, metonimija, kognitivizam, višeznačne lekseme 